\title{
A heuristic methodology to tackle the Braess Paradox detecting problem tailored for real road networks
}

\author{
Saeed Asadi Bagloee ${ }^{\mathrm{a}, 1}$, Avishai (Avi) Ceder ${ }^{\mathrm{b} *}$, Madjid Tavana $^{\mathrm{c}}$ and Claire Bozic ${ }^{\mathrm{d}}$ \\ ${ }^{a}$ Department of Civil and Environmental Engineering, Sharif University of Technology, Tehran, Iran; \\ ${ }^{b}$ Department of Civil and Environmental Engineering, University of Auckland, Auckland, New Zealand; \\ ${ }^{c}$ Department of Information Systems, La Salle University, Philadelphia, PA, USA; ${ }^{d}$ Chicago Metropolitan \\ Agency for Planning (CMAP), Chicago, IL, USA
}

(Received 25 May 2012; final version received 16 March 2013)

\begin{abstract}
Adding a new road to help traffic flow in a congested urban network may at first appear to be a good idea. The Braess Paradox (BP) says, adding new capacity may actually worsen traffic flow. BP does not only call for extra vigilance in expanding a network, it also highlights a question: Does BP exist in existing networks? Literature reveals that BP is rife in real world. This study proposes a methodology to find a set of roads in a real network, whose closure improve traffic flow. It is called the Braess Paradox Detection (BPD) problem. Literature proves that the BPD problem is highly intractable especially in real networks and no efficient method has been introduced. We developed a heuristic methodology based on a Genetic Algorithm to tackle BPD problem. First, a set of likely Braess-tainted roads is identified by simply testing their closure (one-by-one). Secondly, a seraph algorithm is devised to run over the Braess-tainted roads to find a combination whose closure improves traffic flow. In our methodology, the extent of road closure is limited to some certain level to preserve connectivity of the network. The efficiency and applicability of the methodology are demonstrated using the benchmark Hagstrom-Abrams network, and on a network of city of Winnipeg in Canada.
\end{abstract}

Keywords: Braess Paradox detection; heuristic algorithm; genetic algorithm

\section{Introduction}

In 1969, when a new road was constructed in Stuttgart, traffic flow worsened and only improved after the road was torn up; in 1990, when 42nd street was closed in New York City, the traffic flow actually improved instead of the anticipated traffic congestion. These paradoxical phenomena are known as the Braess Paradox (BP). The BP was first recognised by an economist Arthur (Pigou 1920) and it was named after Braess, a mathematician who articulated the phenomena (Braess 1968; Braess, Nagurney, and Wakolbinger 2005). Braess (1968) noted that when a new link is added in a user-optimised network, change in the equilibrium flows might result in a higher cost.

A network consists of links associated with non-decreasing function of flow known as cost. 'Users' unilaterally choose least cost (or shortest) path on the network. The resulting flow is known as User Equilibrium (UE). However, there is a better flow pattern if the users cooperate with each other to make a flow that is best for all the users in the system. 'Best for all users' means that the total costs incurred to all the users is minimum. Such a flow is known as System Optimum (SO). When a new link is added, it is aimed to lower the total cost in the system (SO), but the users pursue a new UE. If we calculate total costs incurred to all the users of this new

*Corresponding author. Email: a.ceder@ auckland.ac.nz 
UE, it might become bigger than the total costs on the previous UE. This counter-expected fact is the BP. The BP is rooted in behaviour of the users; they selfishly choose their own least cost routes without any regard for how their choices may affect others. This is also called selfish routing. If the users decided to collaborate to constitute $\mathrm{SO}$, there would be no place for BP. The difference between total costs incurred in UE and SO is the expense that the users are paying for their selfish behaviour (some pay more some pay less or zero). This expense is coined as price of anarchy (PA).

Interestingly, in 1968 an ecologist with no acquaintance to Braess work coined 'tragedy of commons' (Hardin 1968), which is based on the selfish behaviour of consumers who disregard the scarcity of resources. BP has implications in other disciplines such as Supply Chain, Internet, Electrical circuit, finance and economy (Boyce, Mahmassani, and Nagurney 2005; Nagurney, Parkes, and Daniele 2007; Roughgarden 2005).

Potential BP conditions should make us vigilant when considering road network expansion and also highlight that existing networks may be affected by the BP. Hence, it is worthwhile to investigate whether BP does exist in an existing network, and, if so, which subsets of links cause BP. Let us call the link that individually causes BP a 'Braess-tainted' link. If one finds such Braess-tainted links, the overall traffic conditions will be improved by closing them individually. In some studies, the links that individually cause the BP (Braess-tainted links) are identified (Park 2011 and the references therein). In such studies, two important points are missing: (i) Consider a network in which both links ' $a$ ' and ' $b$ ' are identified as causing BP (Braess-tainted links). Consequently, in two networks, one without link ' $a$ ' and the other one without ' $b$ ', the overall performances would be better than the original network. It is, however, conceivable that a single network without links ' $a$ ' and ' $b$ ' may not perform better compared with the original network. This means that ' $a$ ' and ' $b$ ' individually are Braess-tainted links, but links ' $a$ ' and 'b' combined do not cause Braess Paradox. (ii) Consider a network in which link 'a', 'b' and 'c' are identified as Braess-tainted links. There might be a subset of them, perhaps $\{a, b\}$ or $\{a, c\}$ or $\{b, c\}$ but not necessary all of them $\{a, b, c\}$, whose closures yield maximum performance. We call the effort to find this subset BPD (or the BPD problem).

As real examples introduced earlier indicate that the BP may be found in existing networks. Furthermore, Valiant and Roughgarden (2010) provided the first proof that BP does exist in large size networks (by size we mean number of links), and it is frequent. In theory, BPD is an NP-hard problem (Park 2011; Roughgarden 2001) meaning that it is a very difficult computational effort. It is the very number of identified Braess-tainted links, hence combinatorial feature of the subsets, which makes BPD very intractable. Accordingly, as the size of the network increases, finding an exact solution for the BPD problem becomes impossible (Roughgarden 2001). Therefore, previous works were primarily focused on small size networks (Chung and Young 2010). To our knowledge, the attempt to identify the best combination of Braess-tainted links as described in the definition of our BPD has not been undertaken. Hence, in this study we tackled the BPD problem for large-size networks. While finding exact solution to BPD problem (for large-size networks) is very difficult, we tried to find a good solution via a heuristic approach.

Our methodology is straightforward and has two phases. Phase 1 identifies a set of likely Braesstainted roads. In Phase 2, a search algorithm goes through set of Braess-tainted roads to find a subset of them, whose closure make as much as possible improvement in traffic. The search algorithm consists of two modules: During the first module, the algorithm is run to collect an enriched pool of scenarios. Scenarios are treated as chromosomes in Genetic Algorithm (GA). The first module entails two processes: first, some random chromosomes are generated; second, chromosomes are evaluated to provide the algorithm with some clues to generate better chromosomes. The outcome of the first module is an enriched pool of chromosomes. 
In the second module, chromosomes are mated to breed better generations. At the end of the algorithm, chromosome with maximum improvement in traffic would be the best solution found for BPD problem: Braess-tainted roads who contributed in the chromosome have to be closed. Roads closure may longer the journey of some users due to network disconnection. In our methodology, the extent of road closure is capped to some certain level to preserve connectivity of the network.

The following is a brief outline of the proposed methodology:

- Phase 1: Creating a set of Braess-tainted streets.

- Phase 2: Scenario generation and search algorithm.

- Module 1: Creating chromosomes pool.

- Process 1: Generating initial random chromosome.

- Process 2: Generating advanced chromosomes on the basis of evaluation points.

- Module 2: Mating in the chromosomes pool to breed better solutions.

The efficacy and applicability of the proposed methodology are demonstrated with a large-size network of the city of Winnipeg in Canada. Furthermore, a numerical application of the methodology to a benchmark problem in the literature is presented to delineate the accuracy and the merits of the methodology. The results are promising in a way improvement achieved in Winnipeg was equivalent to construction of a new bridge.

The rest of this paper is organised as follows. Section 2 briefly reviews relevant literature. Section 3 introduces the BPD problem and discusses Phase 1. Section 4 elaborates Phase 2. Numerical results of two case studies are presented in Section 5. Our conclusions and future research directions are presented in Section 6.

\section{Literature review}

BP has intrigued researchers ever since its discovery by Braess in 1968. Nagurney and Boyce (2005) provide a chronological review of such developments in and around discovery of BP. Apart from Transportation, BP has been observed in other fields such as electrical circuits, mechanical networks of springs and strings, and hydraulic systems as well as loss networks and distributed computational systems in computer and electrical science (Korilis, Lazar, and Orda 1999). In Transportation, BP is conceivable in the context of traffic flow and it is not a curious anomaly (Steinberg and Zangwill 1983). In the literature, the BP has been approached from various angles. In the remaining discussion, we try to shed light on important aspects of BP as well as the relevant attempts at BPD in the literature.

Traffic flow is well formulated by the contribution of Wardrop (1952) and Beckman, McGuire, and Winston (1956). Motorists (users) selfishly choose least cost routes or shortest path. This would lead to a traffic pattern known as UE. There is another and better traffic pattern if the motorists unselfishly cooperative with each other when they choose their routes in a way it is best for all the motorists (or entire system); it is called System Optimal (SO) flow (Dafermos and Nagurney (1984); Dafermos and Sparrow 1969; Fisk 1979; Murchland 1970; Sheffi 1984; Steinberg and Zangwill 1983; Stewart (1980)). Traffic flow and, so does BP, is a function of the interaction between the physical network and the travel demand (supply and demand). Hence, this interaction is highly driven by the volume-delay functions (VDFs) associated with the roads. Types of VDFs are also critical. Roughgarden and Tardos (2002) proved that in networks with linear VDFs, the total travel time at UE traffic is $4 / 3$ times greater than the SO flow (at most). This ratio, known as the 'price of anarchy' (PA) is used as a gauge for measuring the severity of BP. The PA was initially coined by Koutsoupias and Papadimitriou (1999) in game theory and measured how the efficiency of a system degrades due to selfish behaviour of its agents. For 
continuous non-decreasing VDFs, PA can be unbounded. For VDF introduced by the bureau of public roads (see Sheffi 1984), PA is capped to 2.15. Nonetheless, networks' topology plays no role in the determination of the PA (Roughgarden 2002; Roughgarden and Tardos 2002).

Roughgarden $(2001,2002,2006)$ has proved that detecting BP is algorithmically difficult, and designing a large general network that is free from BP is a NP-hard problem. Nonetheless, some studies have tackled BPD by some simplifications: Yang and Bell (1998) released the problem as a continuous optimisation problem. They devised the concept of 'network reserve capacity' to avoid BP in designing networks. Inspired by Braess, for example, Korilis, Lazar, and Orda (1999) established some criteria and rule-of-thumb to avoid BP during adding new links. They assumed that all the links are congested. Hagstrom and Abrams (2001) and Abrams and Hagstrom (2006) presented a non-equilibrium traffic flow; compared with UE some users were better-off and no one was worse-off. It is evident that enforcing such traffic flow is a challenge itself.

Yin and Ieda (2002) demonstrated a reliability version of BP. They showed that the occurrence of BP depends on the demand conditions (similar to Pas and Principio 1997). They proposed a bi-level programming formulation in which the reliability paradox can be avoided. There are, however, some concerns with their methods: It is a continuous network design (like Yang and Bell 1998) formulated as a bi-level non-convex problem. Such problems are very intractable to be addressed (even by their heuristic proposed algorithm for a small-sized sample).

Furthermore, traffic flow is outcome of interaction between network supply and travel demand, both of which impact on BP: (i) Any changes in network conditions may lead to (or free of) BP. This includes both hard changes - i.e., the addition or removal of links - and soft changes e.g., changing the controls or signal phasing at an intersection (Aashtiani and Poorzahedy 2004). (ii) Any change in demand may make the network free of BP if it has already been tainted or vice versa (Pas and Principio 1997). Interestingly, it has been established that under some circumstances, the paradox no longer occurs as travel demand increases (Fisk 1979; Nagurney 2010; Nagurney, Parkes, and Daniele 2007; Pas and Principio 1997). Nagurney (2010) proved this phenomenon by deriving a formula that calculated the demand increase that will guarantee that the addition of a new route will no longer increase travel cost, since the new path will no longer be used. The formulation provided by Nagurney (2010) identifies the intervals of travel demand in which the Braess (dis)appears. This formulation is based on the assumption that the VDFs are linear functions (whereas according to Pas and Principio 1997, VDF's parameters have impacts on these intervals). The application of this formulation is limited by the size of network because it involves the calculation of matrix determinant in which matrices represent the size of the network. Computation of determinants for large matrices is not an easy task for which there are some approximation methods (Ipsen and Lee 2006). However, Lin and Lo (2009) have disputed the whole notion. They investigated the BP in the context of time-dependent queues for dynamic traffic assignment (DTA) and showed that queues in different stages have different impacts on system performance.

Recently, the BP in dynamic networks has received considerable attention, mainly in computer science due to acute shortage of internet bandwidth (see Nagurney, Parkes, and Daniele 2007; Zhang, Lam, and Huang 2008 and the references therein). In transportation, the possibility of the paradox existing under dynamic traffic flows with queuing has been studied: Arnott, De Palma, and Lindsey (1993) showed that users' departure time choice behaviour can also cause the paradox; Daganzo (1998) also discussed the paradox conceptually as a 'hypersensitivity' problem arising from the combination of queue spillover phenomena and users' route choice behaviour. In particular, Akamatsu and Heydecker (2003) have studied BPD in the context of DTA for saturated (congested) networks. They establish the necessary and sufficient conditions for BP to occur. They present a method to detect the links whose increase or decrease in capacity can worsen or improve the total travel costs individually. It is evident that Braess Paradox occurrence is not 
necessarily limited to congested networks. To address non-saturated networks, Akamatsu and Heydecker (2003) proposed a heuristic method to reduce them to a saturated network. The results of applications to real networks have yet to be shown. The main disadvantage of this method is ignorance of combinations of the links. In other words, their methodology yields only the individual Braess-tainted roads.

In a different context, Park (2011) has addressed BPD for the 'stable dynamic' network. Stable dynamic is a new model that describes and provides a stable state of congestion. It is different than conventional UE models, and the difference is rooted in the definition the VDF functions. Park (2011) has developed a path-based formulation to tackle BPD. First, the minimum travel time for each origin-destination (OD) pair subject to non-selfishness routing is computed (let us call this 'reference travel time'). Second, the paths whose travel times are less than their respective reference travel times are identified as Braess paths. The formulation is quadratic and non-convex; hence, the available solution algorithm yields local (not exact or global) optimum solutions. Furthermore, the formulation is path-based (not link-based), which is a prohibitive factor in computation time when network size increases. Park (2011) has applied the methodology to a network of one OD, six nodes and nine links. Application to a real size network has yet to be addressed. Askoura, Lebacque, and Haj-Salem (2011) also proposed a path-based methodology to search for the optimal use of paths in the network. The application to a real network has yet to be addressed.

With respect to nature of the $\mathrm{BPD}$, which is finding the combination of links that cause $\mathrm{BP}$, the main disadvantage of path-based approaches is how to identify Braess links within Braess paths. Even if there is an efficient and exact algorithm that yields Braess path(s) to be closed, the only way is to find link(s) on the Braess path(s) for closure, which is a new challenge.

Traffic information advisories are effective in managing the motorists' perception of traffic (Bagloee, Ceder, and Bozic 2013; Bazzan and Klugl 2001). This can help traffic authorities to mitigate the BP's impacts. Roughgarden (2005) investigated the positive results for three different options: (i) extending network's capacity, (ii) routing a small portion of the traffic centrally and (iii) influencing network users through the use of tolls and taxation. The paper recommends the use of option (ii) to mitigate the impacts of BP, although the paper does not discuss the feasibility of such initiatives. Furthermore, Cole, Dodis, and Roughgarden (2006) advocate adjusting network capacity rather than introducing toll system in the quest to address BPD.

According to the literature review:

- In theory and in practice, the likelihood of BP occurrence is high.

- Any changes in the network from signal timing to adding new capacity could lead to emergence (or removal) of BP.

- BP is not only a function of the network but is also sensitive to travel demand conditions.

- The severity of BP is highly dependent on the type of VDFs, and it can be extremely high.

- Detecting BP in large-size networks is very difficult. There are some attempts to find individual links causing BP. The application of such methods to large size networks has yet to be investigated. Nonetheless, what is most important is to find a combination of such links that collectively cause BP. To our best knowledge, this issue has not been addressed in the literature.

- A feasible approach to mitigate BP's impact is via adjusting network capacity. The idea of BPD emerges here. Are there any combination of roads in the existing network cause the $\mathrm{BP}$. If one can find them, their closure results in a BP free (or less BP) network in which the overall network performance will improve.

- The literature has always described the Braess Paradox phenomenon with titles such as paradox, counter-expected, counter-intuitively, surprising, interesting. Furthermore, some 
studies demonstrate conflicting results. The work of Lin and Lo (2009) versus Nagurney (2010), Nagurney, Parkes, and Daniele (2007), Pas and Principio (1997) and Fisk (1979) contain such conflicting results. This means that still there is a long way to go before we fully understand the Braess phenomena.

The literature review indicates that the BPD in the conventional context of Wardropian UE with fixed (static) and exogenous travel demand is very intractable. When the aim is to address BPD for a real size network, the BPD problem becomes more complex. Extending the current research format to other aspects of BP, such as within DTAs, stable dynamic or path-based approaches are left for further studies.

\section{Creating a set of Braess-tainted streets (Phase 1)}

UE traffic may be expressed as follows (Sheffi 1984):

$$
\begin{gathered}
\text { (UE) Minimizing } \quad \mathrm{UE}\left(v_{\ell}\right)=\sum_{\ell \in L} \int_{0}^{v_{\ell}} t_{\ell}(x) \mathrm{d} x \\
\text { S.t. } \sum_{p \in P_{\text {od }}} h_{p}=D_{o d} \quad \forall(o, d) \in Q \\
h_{p} \geq 0 \quad \forall p \in P_{o d}, \quad \forall(o, d) \in Q \\
v_{\ell}=\sum_{(o, d) \in Q} \sum_{p \in P_{o d}} h_{p} \delta_{\ell, p} \quad \forall \ell \in L
\end{gathered}
$$

where $\operatorname{UE}(\cdot)$ is the objective function that depends on traffic volume $v_{\ell}$ on link $\ell \in L$ in which $L$ is set of links, $t_{\ell}(\cdot)$ is the VDF of link $\ell \in L, h_{p}$ is the traffic flow on path $p \in P_{o d}$ from origin $o$ to destination $d$. Set of all paths between $o$ and $d$ is denoted by $P_{o d}$ and $Q$ is a set of OD pairs. $D_{o d}$ is the travel demand from $o$ to $d . \delta_{\ell, p}$ is 1 if link $\ell$ belongs to path $p$ and 0 , otherwise.

The UE formulation simulates behaviour of users who are trying to find least-cost routes selfishly. Solving UE problem is known as performing 'traffic assignment'. The objective function of Equation (1) is hardly interpretable. If we replace the objective function in UE formulation to total travel cost incurred to all users, we will get SO traffic pattern:

$$
\text { (SO) Minimizing } \mathrm{SO}\left(v_{\ell}\right)=\sum_{\ell \in L} t_{\ell}\left(v_{\ell}\right) \cdot v_{\ell}
$$

s.t.: Equations (2)-(4).

Figure 1(a) depicts a network that consists of one OD pair with six users representing the demand, and connected by two paths and four links. The VDFs are also shown.

The total travel time on the network is 498. A new highway is constructed as Link 5 to improve the traffic flow as shown in Figure 1(b). However, counter-expectedly, the total travel time increases to 552, which is BP. The occurrence of BP is rooted in the fact that the new highway is intended to reduce the SO function (Equation (5)), but the traffic flow is distributed according to UE function (Equation (1)). Therefore, the ensuing traffic flow (UE solution) does not necessarily reduce the total travel time (a detailed discussion is provided by Fisk 1979; Sheffi 1984). 
(a)


Volume Delay Functions (VDF)

$t_{1}(x)=t_{2}(x)=x+50$

$t_{3}(x)=t_{4}(x)=10 x$

$t_{5}(x)=x+10$

$t_{5}^{\prime}(x)=t_{5}^{\prime \prime}(x)=x+10$

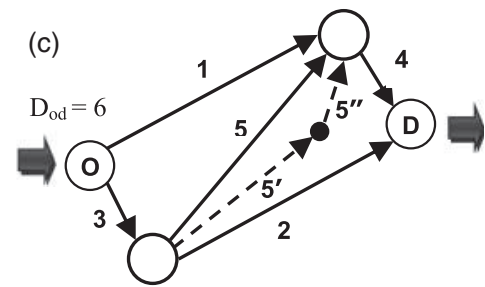

Figure 1. Braess Paradox (BP): (a) A network free of BP, (b) BP occurs; removing link 5 yields a better traffic, (c) BP occurs; removing either links $\left(5,5^{\prime}\right)$, or $\left(5,5^{\prime \prime}\right)$, or $\left(5,5^{\prime}, 5^{\prime \prime}\right)$ yields a better traffic.

In the first phase of the methodology, we tried to identify roads like link 5 in the network presented in Figure 1(b) whose individual closures reduce travel time. Such roads are called Braess-tainted. The idea is simple: if we start testing the removal of all links in Figure 1(b) we will figure out that link 5 is a Braess-tainted link. The same idea is applied to large-size network; given a network and a link, traffic assignment is carried out for networks with and without that link. The total travel time for both the assignments are calculated, if the total travel time for the network without that particular link is less, the link will be identified as Braess-tainted link. Let us denote the set of Braess-tainted roads as $S$ :

$$
S=\left\{\forall s \in L \mid I_{s}=T_{0}-T_{-s}>0, \quad \text { s.t. Equation (7) }\right\}
$$

where $T_{0}$ is the total travel time on the full-network, or Do-Nothing scenario, $T_{0}=\sum_{\ell \in L} t_{\ell}\left(v_{\ell}\right) \cdot v_{\ell}$ ( $v_{\ell}$ from Equations (1-4)); $T_{-s}$ is the total travel time on network without road $s \in L: T_{-s}=$ $\sum_{\ell \in L_{-s}} t_{\ell}\left(v_{\ell}\right) v_{\ell}$ ( $v_{\ell}$ from Equations (1-4), wherein $L$ is set $L_{-s}=L-\{s \in S\}$, in which $I_{S}$ is called 'intrinsic point' of $s \in S$ (see also discussion related to Equation (11)).

The closure of roads should not compromise connectivity of the network. The entry to set $S$ is subject to passing a connectivity filter to make sure that for every OD there is an operational path(s). For large-size networks where the number of OD pairs and paths are high, there would no easy way unless traffic assignment is carried out to obtain travel time for each OD to setup connectivity measures. Based on Chen et al. (2002), the connectivity filter is defined as follows: the increase in $T_{-s}(o, d)$ travel time between every OD pair after closing road $s \in L$ is capped to a certain level depending on $T(o, d)$ corresponding travel time on the full-network. This certain level is specified by coefficient $\alpha_{t}$ as an index for level of service (LoS) due to roadclosure. The connectivity filter may be written as follows:

$$
\begin{aligned}
T_{-s}(o, d) & \leq \alpha_{t} T(o, d) \quad \forall(o, d) \in Q \\
\alpha_{t} & =4.171 . T(o, d)^{-0.343}
\end{aligned}
$$

where $\alpha_{t}>1$ is the outcome of a simple regression with two assumed entries: (i) for those on short trips with $5 \mathrm{~min}$ travel time, maximum acceptable travel time due to any road closure is $12 \min$ (i.e. $T(o, d)=5, T_{-s}(o, d)=12$ ). (ii) For those on long trips (say $T(o, d)=40$ ) only a 


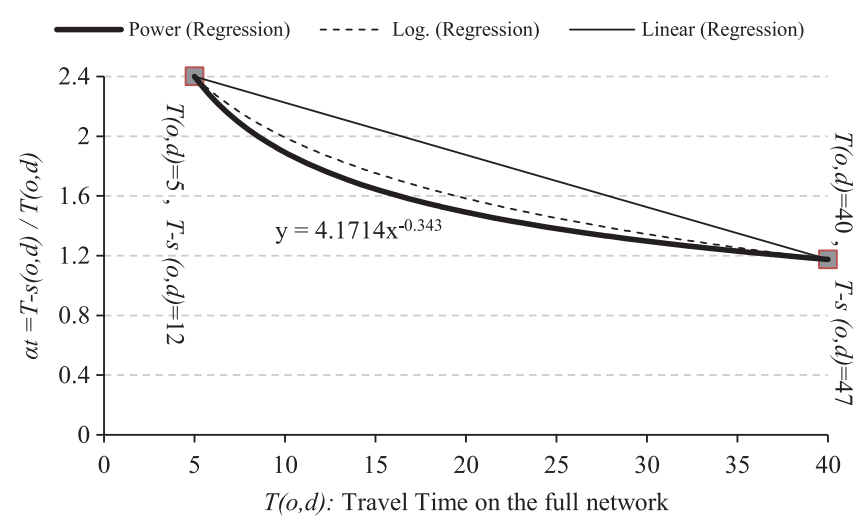

Figure 2. Various graphs for $\alpha_{t}$ level of service index for connectivity filter in road closure.

marginal increase in travel time is acceptable ( $\operatorname{say} T_{-s}(o, d)=47$ ). The rationale behind the above arrangement is based on the fact that motorists who experience more travel time are less patient about increased delay on their journey (Bagloee, Ceder, and Bozic 2013). The regression used is power, which gives more convexity for $\alpha_{t}$ compared with other types such as linear, logarithmic and exponential. Figure 2 depicts various graphs of $\alpha_{t}$ (LoS index) with the two entries. The convexity of the LoS index complies with the results of LoS analysis in Chen et al (2002). More convexity implies that marginal impact of change in travel time is felt much strongly in short trips compared with long trips (Bagloee, Ceder, and Bozic 2013; Chen et al. 2002).

It is important to note that theoretically, set $S$ cannot encompass all road-for-closure in exact solution to BPD problem. For instance, if a parallel route to link 5 (in Figure 1(c)) exists, in case of removing link 5, the parallel route would play a role of link 5 and will carry its traffic volume. Therefore, the intrinsic point of link 5 will be 0 and after all no link is identified as Braess-tainted (see links $5^{\prime}, 5^{\prime \prime}$ as parallel route to link 5 in Figure 1(c)). Nonetheless, this deficiency can be compensated in the context of complexity involved with BPD and premium of finding good (not necessary exact) solutions in real-size networks.

Road closure testing is a widely used method in studies of network reliability, vulnerability and robustness. Such studies emerged in the past decade due to some unprecedented events (natural disaster or terrorist attacks). Hence, it is worthwhile to rank the network elements to allow special attention to top tier elements needed to keep the network up and running in the case of unexpected events. Analogues to Equation (6), the existence and absence of network elements are evaluated and based on various characteristics they are quantified. The network elements are then ranked based on the quantified measures (Jenelius 2010; Nagurney and Qiang 2008; Novak, Sullivan, Scott 2012; Poorzahedy and Bushehri 2005; Sullivan et al 2010). One may constitute the Braesstainted set by including the roads in the lowest tier of the ranked list. In our methodology, the lowest tier contains the links who have positive intrinsic points $\left(I_{S}\right.$ in Equation (6)).

As noted before, the literature provides alternative methods to find individual Braess-tainted links, which could be used here in phase 1 of our methodology to develop set $S$. What is important is the combination of the road closures (Bagloee and Tavana 2012). For instance, if some roads become closed, the ranking order of the remaining roads ought to change.

Therefore, in the following discussion a subset of Braess-tainted roads $\left(S^{\prime} \subseteq S\right)$ that yields maximum benefit is sought. The goal is to save total users' travel time. The BPD problem may be written as maximising the time saved on road network $L_{-S^{\prime}}=L-S^{\prime}, S^{\prime} \subset L$. The problem is subject to UE flow and connectivity criteria. If one considers $Z\left(V_{S^{\prime}}\right)$ as the benefit of closing 
subset $S^{\prime}$ expressed in terms of the amount of users' time saved, the BPD problem may be written as follows:

$$
\text { (BPD) } \underset{S^{\prime}}{\operatorname{Maximize}} Z\left(V_{S^{\prime}}\right)=T_{0}-\sum_{\ell \in L_{-S^{\prime}}} t_{\ell}\left(v_{\ell}\right) \cdot v_{\ell}
$$

s.t. $v_{\ell}$ from Equation (1 to 4), Equation (7).

Phase 1 provides a set of Braess-tainted roads $(S)$ to which $S^{\prime}$ in Equation (9) is a subset $\left(S^{\prime} \subseteq S\right)$.

\section{Hybrid scenario generation and search algorithm (Phase 2)}

Phase 2 is devised to find $S^{\prime}$ by employing GA means and terminology in accordance with Equation (9). In addition to GA, there are other complicated evolutionary methods that could be used. However, given the complexity of the BPD per se, we adopted GA in its widely known conventional configuration (Holland 1975) to allow us to devote much of the discussion to the BPD problem and the solution. Scenarios are coded as chromosomes. A chromosome is a string of genes corresponding to the Braess-tainted roads (set $S$ ). A gene contains a binary values (1: if the corresponding road has to be closed and 0 : otherwise). Phase 2 is an iterative algorithm consisting of two successive modules:

- In Module 1, a pool of chromosomes is generated. For this module, termination criterion is the maximum number of iterations: $i_{\max }$. The number of iterations must be able to cover the solution space. The extent of the solution space is demarcated by the number of Braesstainted roads $(n=|S|)$. Hence, $i_{\max }$ depends upon $n$. Since at each iteration, the chromosome contains at least one filled gene, and to give each Braess tainted road at least one chance to contribute in a chromosome, we set $i_{\max }=|S|$.

- In Module 2, chromosomes in the pool are mated to breed better chromosomes. Parent selection and mating operations are repeated until no better chromosomes are generated. In Section 4.2.3, the termination criterion for Module 2 is elaborated.

Throughout Phase 2 (in Modules 1 and 2) in each iteration (i) a chromosome $\left(S_{t}\right)$ is generated. Traffic assignment procedure is then carried out to compute the benefit of the chromosome (saved time, or $Z\left(V_{S_{i}}\right)$ of Equation (9)) as an evaluation index. If the chromosome satisfies the connectivity criteria (Equation (7)), we assign $Z\left(V_{S_{i}}\right)$ to chromosome $S_{i}$ as the scenario benefit:

$$
Z_{i}=\left\{\begin{array}{cc}
Z\left(V_{S_{i}}\right) & \text { Equation (7) holding } \\
0 & \text { otherwise }
\end{array}\right\}
$$

Genes (or Braess-tainted roads) are also measured by $I_{i, s}$, which is the point collected by Braesstainted (or gene) $s \in S$ at iteration $i$ :

$$
I_{i, s}=\left\{\begin{array}{cc}
g_{i, s}, & \sqrt{Z_{i} . I_{s}} Z_{i}>0 \\
0 & \text { otherwise }
\end{array}\right\} \quad \forall s \in S
$$

where $g_{i, s}$ is 1 if gene $S$ has contributed in chromosome of iteration $i$ and 0 , otherwise; $I_{s}$ is the point collected by a gene in a chromosome that there is no other genes contributed, which we call it intrinsic point:

$$
I_{s}=Z\left(V_{s}\right) \mid s \in S
$$

Points in Equation (11) are bound to geometric mean of intrinsic point $\left(I_{S}\right)$ and chromosome's benefit $\left(Z_{i}\right)$. The reason is to eliminate the possibility of assigning high points to weak genes (low $I_{S}$ ) who by chance contributed to strong chromosome (high $Z_{i}$ ) and vice versa. 


\subsection{Module 1; creation of chromosomes pool}

Module 1 consists of two processes. First, a limited number of purely random chromosomes are generated. Second, based on the evaluation of chromosomes more advanced chromosomes are generated.

\subsubsection{Generating initial random chromosome (Process 1)}

Initial solution(s) is(are) required to launch any iterative solution-finding algorithm. Solutions represent characteristic of surrounding area within solution spaces and in heuristic algorithms, next move is based on topographical knowledge of the solution space. Hence, in the absence of any pre-knowledge, initial solutions must be randomly distributed on the solution space. We ensure that with significant confidence every road $s \in S$ will appear at least once in the initial chromosomes. First question is how many initial solutions are required which depends upon how many roads contribute in the chromosomes. It is expected that a portion (not all) of Braess-tainted roads would contribute in good chromosomes. This portion is called Chromosome capacity denoted by $\bar{n}, 1<\bar{n}<n$; that can be interpreted as number of roads to be closed in the best chromosome. Because there is no clue for $\bar{n}$, it shall be specified via sensitive analysis. Now let $q$ be the number of initial chromosomes. Probability of not having a road $s \in S$ contributed in one initial chromosome is $(n-\bar{n}) / n$. Therefore, probability of having a road $s \in S$ contributed in non of the initial chromosomes is $((n-\bar{n}) / n)^{q}$. Now probability of having a road $s \in S$ has contributed at least once in $q$ initial chromosomes is $1-((n-\bar{n}) / n)^{q}$. For this probability, we consider a significant confidence and hence number of initial chromosome $(q)$ can be identified. In theory of probability, significant confidence is regarded as 0.95 chance or above. Thus:

$$
1-\left(\frac{(n-\bar{n})}{n}\right)^{q}=0.95
$$

For instance in Winnipeg case study, described below, $n=334$ for various values of chromosome capacity of $\bar{n}=\{3,50,100\}$ and number of initial chromosomes would be $q=\{334,18,8\}$, respectively.

Thus far, given the chromosome capacity $(\bar{n})$, the size of initial chromosomes $(q)$ is identified. In the following discussion, we explain how to fill out these $q$ chromosomes.

In the quest to build up chromosome, there are two important points:

(1) Initial chromosomes are supposed to lead to good solutions. Hence, inferior chromosomes are excluded in initial chromosomes. Therefore, chromosomes have to pass a qualification entrance test: Chromosomes whose benefits are greater than the maximum intrinsic point are qualified to become an initial chromosome:

$$
\text { Benefit qualification : } Z_{i}>\max \left(I_{s}\right) \quad \forall s \in S, \quad i=1, \ldots, p_{b}
$$

(2) We are uncertain about the exact value for chromosome capacity $(\bar{n})$. Hence, in each iteration throughout Phase 2 including the current process, we let the capacity of chromosome fluctuate around $\bar{n}$. Consider $1<\bar{n}_{i} \leq n$ as the capacity of chromosome $i, 0<\rho<1$ is a random variable and $[\cdot]$ is a function that yields the integer part of its argument:

$$
\bar{n}_{i}=[2 \rho \bar{n}]
$$

Note that $2 \rho \approx 1$, so the average value of $\bar{n}_{i}$ will be $\bar{n}$. 
Now, the way is paved to produce $q$ initial solutions (or chromosomes).

- Repeat the following steps for iteration $1 \leq i \leq q$.

- Specify $\bar{n}_{i}$ chromosome's capacity (Equation (15)).

- Assign random numbers to every $\operatorname{road}\left(0<\rho_{s}<1, \forall s \in S\right)$.

- Sort the roads based on random numbers ascendingly.

- Make a chromosome by picking $\bar{n}_{i}$ number of roads from top of the sorted list.

- Carry out traffic assignment for this chromosome.

- Does the chromosome satisfy connectivity (Equation (7)) and benefit qualification (Equation (14))? Was not repeated in preceding iterations?

- If all are yes: Compute benefit of chromosome (Equation (10)) and points of roads (Equation (11)) and proceed for next iteration.

- Otherwise: resume all these efforts again.

The outcome of this process is $\Phi$, which is pool of chromosome with $q$ entries.

\subsubsection{Generating advanced chromosomes on the basis of points (Process 2)}

The initial chromosomes $(\Phi)$ and associated information $\left(Z_{i}, I_{i, s}\right)$ configures the base to generate more advanced chromosomes. In this process, the roads are selected on a merit basis, which is the number of points collected in the preceding iterations. The preceding chromosomes are sorted in descending order based on their benefits $\left(Z_{i}\right)$. Points obtained by road $s \in S$ in the $c$ th chromosome in the sorted list is renamed by $I_{c, s}^{\prime}$. Merit index of roads is defined as the number of points collected by roads in $q$ top chromosomes in the sorted list combined with intrinsic point $\left(I_{s}\right)$. Consider $M_{i, s}$ as the merit index of road $s \in S$ computed for iteration $q+1 \leq i \leq i_{\max }$ :

$$
M_{i, s}=I_{s} \rho^{\left(1-i / i_{\max }\right)}+\sum_{c=1}^{q}\left(I_{c, s}^{\prime} \rho^{\left(1-i / i_{\max }\right)}\right)
$$

where a random number $(0<\rho<1)$ makes combination of the points stochastically (or volatile). Power terms of random $\left(1-i / i_{\max }\right)$ makes early iterations (low $i$ ) highly volatile. Volatility gives chance to every road to contribute in chromosomes to show its merit. As the process proceeds $\left(i \rightarrow i_{\max }\right)$, the volatility becomes less and only roads with solid merits will get such chances. Why points collected based on merely $q$ top chromosomes is complied in merit index, because, as discussed all roads $(s \in S)$ are likely to be captured at least once. Furthermore, the presence of intrinsic point $\left(I_{S}\right)$ in Equation (16) gives such chance to good genes who has not probably contributed in $q$ top chromosomes $\left(I_{c, s}^{\prime}=0\right)$.

The process is completed by sorting roads in descending order based on merits and to pick up $\bar{n}_{i}$ roads from top of the list. If this chromosome has been generated in preceding iterations, the algorithm applies 'Mutation' to amend the chromosome. Mutation is an important feature of GA to prevent stagnating at local optima. The mutation is applied as follows: two genes with contents of 0 (not contributed in the chromosome) and 1 (has contributed in the chromosome) are randomly selected and their contents are swapped.

Like before, the chromosome is evaluated. The traffic assignment is carried out to evaluate the connectivity criteria and to calculate the benefit and point indices in Equations (10) and (11).

The outcome of this process is $\Phi$; a pool of chromosome that is passed to the next module.

\subsection{Breeding the chromosomes pool to get better solutions (Module 2)}

In Module 1, chromosomes based on roads' merit were generated. According to GA, these chromosomes ought to breed better generations be properly combined (mated). In Module 2, such 
breeding is operated. It is an iterative operation. In each iteration, to enhance the quality and the diversity of the next generations, two strong and ordinary chromosomes are selected and then mated.

\subsection{1. $\quad$ Selecting (parents) chromosomes}

First, chromosomes in the pool are sorted in the descending order based on benefits $\left(Z_{j}, j=\right.$ $1 \ldots|\Phi|)$. Let us denote the sorted list as $\Phi^{\prime}$. A random number is applied to break $\Phi^{\prime}$. The chromosome at the breaking point is assumed to be an ordinary chromosome. Consider $C_{1}$ as the position of the ordinary chromosome in $\Phi^{\prime}$ :

$$
\begin{gathered}
C_{1}=\left[x\left|\Phi^{\prime}\right|\right] \\
x=\rho, 0<\rho<1
\end{gathered}
$$

where $x$ implies the location at which $\Phi^{\prime}$ is broken (i.e. $x\left|\Phi^{\prime}\right|$ ). Strong chromosome shall be chosen from a range in $\Phi^{\prime}$ from first top chromosome down to the chromosome at breaking point $\left(C_{1}\right)$. Consider $1 \leq C_{2}<C_{1}$ as the position of a strong chromosome in $\Phi^{\prime}$ :

$$
C_{2}=\left[(1-x) \rho C_{1}\right]
$$

where inclusion of $(1-x), \rho$ are random numbers with an average of 0.5 that push $C_{2}$ to position of $0.25 C_{1}$. The presence of $(1-x)$ in Equation (19) makes $C_{2}$ more inclined to top of $\Phi^{\prime}$ list, should the ordinary chromosome is chosen from bottom of the sorted list (i.e. $x \rightarrow 1 \Rightarrow(1-x) \rightarrow 0$ ).

\subsubsection{Mating operations}

Now the (parent) chromosomes are mated to breed a new chromosome as follows:

- Repeat the following steps for each gene from 1 to $n=|S|$ :

- Generate a random number $\rho$.

$\circ$ if $(1-x) \rho<x$ then the gene is inherited from strong chromosome, otherwise from ordinary chromosome.

The same rationale is applied in the mating operations: The new chromosome inherits genes from the parents randomly, but favours the strong chromosome. If the ordinary chromosome is too weak $(x \rightarrow 1)$, then $(1-x) \rightarrow 0$ and probably we have $(1-x) \rho<x$, which means gene will be copied from strong chromosome.

Like before, if the new chromosome already exists in the chromosome pool, the mutation procedure is applied to amend the chromosome. The iteration is completed by traffic assignment connectivity appraisal and evaluations.

\subsubsection{Termination criteria for mating operations (in Module 2 of Phase 2)}

Parent selection and mating operations are repeated until no better chromosomes are generated for some successive iterations. After the best (so far) solution is found, one may want to continue the operation until for each filled gene there is one chance to be replaced with a better gene. For instance, in the Winnipeg case study, the best solution found contains 62 roads (filled genes). Therefore, we carried out the operation for 62 more iterations during which no better solution was found.

Figure 3 graphically outlines the methodology. 


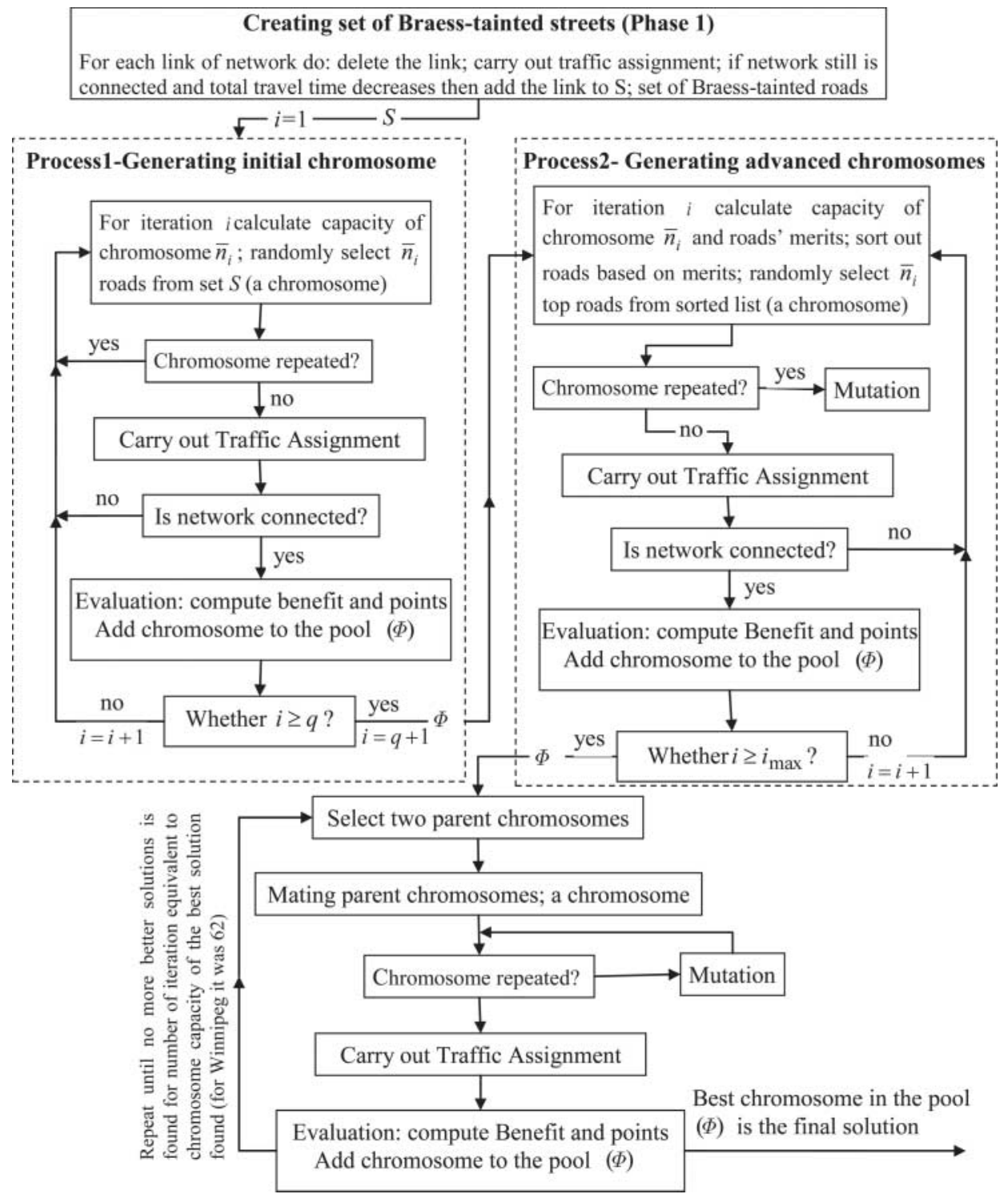

Figure 3. Outline of the methodology.

\section{Numerical examples}

The methodology, written in Visual Basic (VB), is linked to MS-Excel (for input/output interface) and EMME/3 (for the traffic assignment). To efficiently handle the huge amount of data, the program is linked to a MS-ACCESS database. Integration and synchronisation of VB, MS-Excel, EMME/3 and MS-ACCESS have streamlined the application of the methodology with respect to efficient computation time and being a user-friendly platform. A PC with a ' $1.86 \mathrm{GHz}$ CPU and 2.00 GB of RAM' is used. We first applied the methodology to benchmark the network of Sioux Falls, which consists of 24 nodes, 76 links, 528 non-zero O-D pairs and 3600 trips as demand (BarGera 2010). In the first phase, no link was identified as Braess-tainted (i.e. $T_{0}-T_{-\ell} \leq 0 \mid \forall \ell \in L$ ). 
Nonetheless, it does not mean that the network is free of BP and the Sioux Falls is still open for further studies. Following are the numerical results of two other networks.

\subsection{Winnipeg}

Winnipeg data are a standard database distributed by Inro, the EMME/3 developers (INRO 2007). The data are already coded and made available by INRO once the EMME/ 3 software is installed, which makes its use widespread and easily available for practitioners and researchers for further analysis. Furthermore, the raw data are freely available on the Internet too (Bar-Gera 2012). The network consists of 154 zones, 4420 non-zero oD pairs and 56,219 hourly trips as demand. The total travel time for the 'Do-Nothing' scenario is $T_{0}=814,678$ ( $\left.\mathrm{min}\right)$. The network consists of 2975 links. Thus, for Phase 1, in accordance to Equation (6), traffic assignments were executed 2975 times and a set of Braess-tainted roads was developed with 334 entries. The operations took $6 \mathrm{~h}$. The Braess-tainted set $(|S|=334)$ is passed to Phase 2 .

\subsubsection{Module 1 (of Phase 2), Winnipeg}

In Module 1, two parameters must be specified: number of iterations $i_{\max }$ pertaining to Module 1 of phase 2 and chromosome capacity $\bar{n}$. At each iteration chromosome contains at least one filled gene $(\forall s \in S)$. To give every Braess-tainted road at least one chance to contribute in a chromosome, we set $i_{\max }=|S|=334$. Given $i_{\max }=334$ we applied Module 1 to a wide range of chromosome capacity $(\bar{n})$. Based on the discussion presented for Equation (13), the lower bound for chromosome capacity would be $\bar{n}=3$, where the maximum size of initial chromosome becomes identical to number of iterations $\left(q=i_{\max }=334\right)$. This means that the maximum possible for $q$ is equal to 334 and it corresponds to $\bar{n}=3$. The upper bound is specified via a sensitive analysis as follows: In order to flatten unusual results due to the presence of random numbers in Module 1, we repeat each run 100 times and the average results are reported. Figure 4(a) depicts the benefit of best solutions found $\left(Z_{i}\right)$ versus incremental values of $\bar{n} \geq 3$. The horizontal axis of Figure 4(a) terminates at $\bar{n}=100$ (upper bound), where the benefits have a continuous negative slope. Almost a symmetric dome shape in Figure 4(a) indicates that the algorithm of Module 1 behaves rationally. With respect to Figure 4(a), we choose $\bar{n}=50$ for further analysis while according to Equation (13) the number of initial chromosome is computed as $q=18$. In the above quest, almost half of the iterations became null either by breaching connectivity criteria or by yielding negative benefits at the end. To compensate for null iterations, we consider $i_{\max }=2 \times 334=668$. Hence, the outcome of Figure 4(a) is chromosome capacity, number of initial chromosomes, and the maximum number of iterations for Module $1\left(\bar{n}=50, q=18\right.$ and $\left.i_{\max }=668\right)$. Therefore, we run Module 1 with these new parameters. Figure 4(b) shows the behaviour of the algorithm over successive iterations for one run. In Figure 4(b):

- To provide a contrast of the intrinsic point with trend of the results, the first 334 records are intrinsic points. Hence, the horizontal axis entails 1002 records (i.e. $1002=334+668$ ).

- The median part $(i=335, \ldots, 540)$ is highly chaotic, reflecting Process 1 dedicated to generating random initial chromosomes.

- Out of 1002 records, 251 records whose benefits becomes zero: $Z_{i}=0$ in Equation (10) breached the connectivity criteria (Equation (7)). The majority of these records fall in the range of $i=669, \ldots, 774$ after which the algorithm refocused and began to create better chromosomes.

- Out of 1002 scenarios in 142 scenarios, the connectivity criterion is respected but closing their respective roads will not improve the overall network performance: $\left(Z_{i}<0\right.$ in 



Figure 4. Numerical results of testing Winnipeg, Canada case study: (a) Analysing a range of chromosome capacity, $i_{\max }=334$, each record represents average results of 100 runs, (b) trend of solutions found in Module 1, and (c) trend of solutions found in Module 2. 
Equation (10)). This means that the collection of Braess-tainted roads does not demonstrate Braess Paradox characteristics together.

- At the end of Module 1, the best found solution comprises 65 roads whose closure yields the benefit of 5964 saved time in minutes. A pool of chromosome $(\Phi)$ with 609 entries is put forwarded for Module 2.

\subsubsection{Module 2 (of Phase 2), Winnipeg}

Selection/mating operations are applied to the inhabitants of the chromosomes pool $(\Phi)$. Figure 4(c) shows an upward trend of results pertaining to Module 2 of Phase 2. The lower bound of the $y$-axis in Figure 4(c) represents the best solution in the previous module (5964).

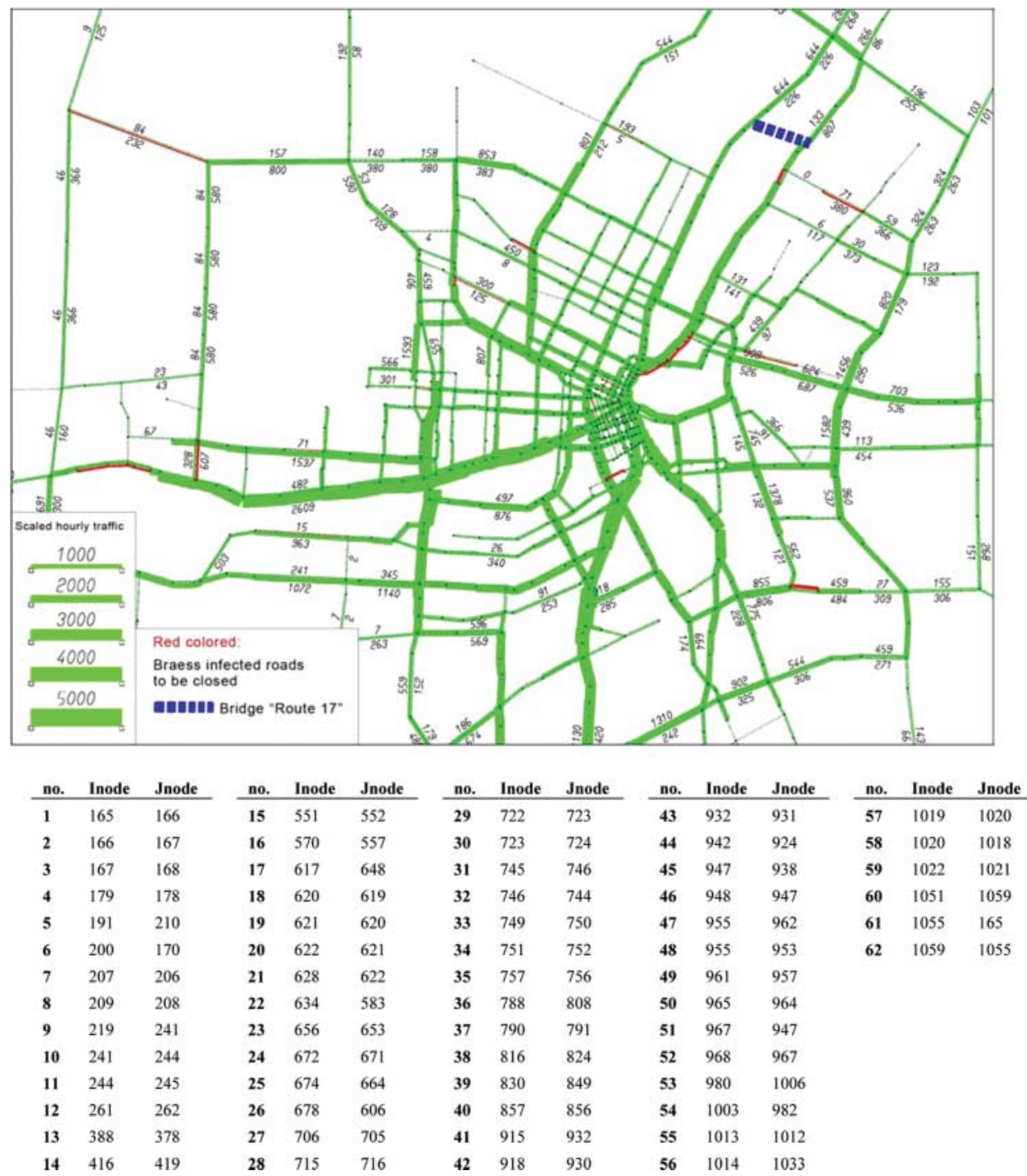

Figure 5. Winnipeg case study. Hourly traffic volume in the existing scenario, 62 links found for closure as Braess infected roads and location of Bridge 'Route 17'. 


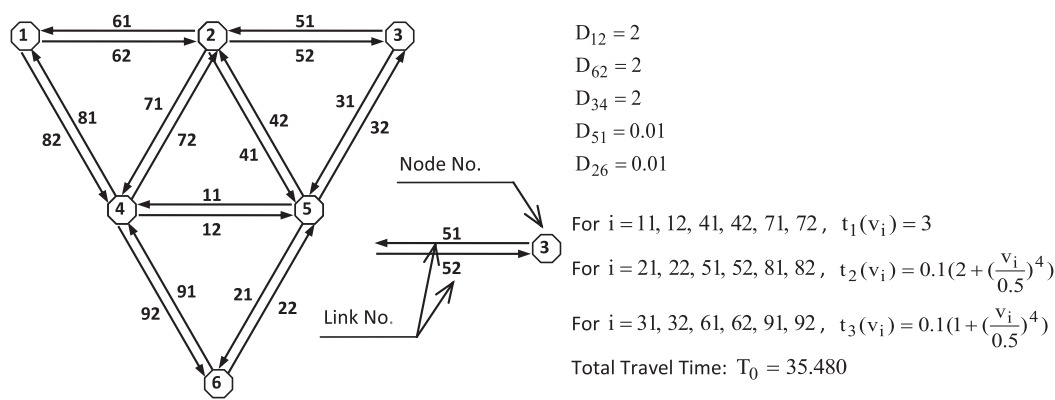

Figure 6. A BP-contaminated sample similar to Hagstrom-Abrams benchmark.

Now in the current module, the best found solution $\left(Z_{i}=6989\right)$, with 62 roads for closure was obtained at trial 204. The algorithm is repeated 62 more iterations from trials 217 to 278 during which no improvements are seen (the rational is explained in Section 4.2.3). These 62 roads are shown in Figure 5. The total length of these roads is 32.79 mi-lanes or $1.01 \%$ of total existing mi-lanes. It is equivalent to closing 8.19 miles of a two-way street with two lanes in each direction. The availability of the Winnipeg data set as noted earlier along with the best solution found in this study shown in Figure 5 can make Winnipeg a benchmark for further studies in Braess Paradox detection.

It is useful to understand the road construction equivalent benefit of the road closure $\left(Z_{i}=6989\right)$. The Winnipeg Database used here reflects the city's network in the late 1980s (INRO 2007). In 1990, bridge 'Route 17', known as 'Chief Peguis Trail', (connecting nodes 178 and 878 over Red river) with 1 mile long and two lanes/direction was inaugurated. The location of the bridge is depicted in Figure 5. Coding the bridge in the model followed by a traffic assignment showed a 5903-min decrease in the total travel time. This saving versus 6989 resulting from road closure indicates that the idea of road closure deserves to be taken seriously. Road closure is a supply-management tool with no cost, whereas constructing a bridge is a huge civil construction project, requiring considerable time, money, as well as social and environmental impacts.

\subsection{A case study similar to Hagstrom-Abrams example}

The best way to evaluate the algorithm is to see how close the identified solutions are to the exact solution. Inspired by Hagstrom and Abrams (2001), we developed a BP-contaminated network with 18 links shown in Figure 6. Phase 1 identified six Braess-tainted roads $(n=|S|=6)$ as $S=\{61,81,52,32,21,92\}$. Enumeration revealed the exact solution to be $S^{*}=\{61,52,32,92\}$, $\left(\bar{n}=\left|S^{*}\right|=4\right)$, which yields a benefit of 2.666 . The enumerations of all permutations $\left(2^{18}=\right.$ $262,144)$ took 3 days and $7 \mathrm{~h}$. With respect to the small size of the network, we challenged the methodology by: (i) excluding Module 2; (ii) using $i_{\max }=10$ rather than $2 \times 6=12$. We ran the methodology over a range of chromosome capacity: $\bar{n}=\{3,4,5,6\}$ for which according to Equation (13) we have $q=\{4,3,2,1\}$. Each run was repeated 100 times and the expected benefits became $\{1.869,2.401,2.383,1.873\}$ while the frequency of reaching the exact solution was $\{0,51,28,22\}$. Hence, $\bar{n}=4$ is the best performing chromosome capacity at which the probability of achieving the exact solution is more than 50\% (even though Module 2 is excluded).

\section{Conclusions and applications}

This study addresses BPD's problem in actual road networks by identifying roads for closure to improve traffic flow. The literature review revealed that BP is widespread in the real world and 
it is highly difficult to detect. A heuristic methodology consisting of two phases is proposed. In phase 1 inspired by the Braess example, a set of Braess-tainted roads is identified. In phase 2, a heuristic search engine based on GA is launched over the Braess-tainted roads to find a subset of roads whose closures yield maximum improvement. Phase 2 includes two modules. In the first module, an affluent-enriched pool of scenarios (or chromosomes) is produced. In the second module, chromosomes in the pool are mated to breed better generations. As for numerical tests, we used network data of Winnipeg with 152 zones. Final solution turns out to be closure of 62 links which resulted in 6989-min saved time. This was equivalent to constructing 1 mile long bridge.

We developed a small network with 18 roads to see whether the methodology is able to find the exact solution (The exact solution was already found via enumeration). The results showed that the probability of finding the exact solution was above $50 \%$.

In this study, we assumed that travel demand is an exogenous and fixed input. Hence, it is evident that the application of this methodology to various scenarios of travel demands is required before proposing a list of roads for closure. It is also imperative because, as the literature review suggests, the (dis)appearance of Braess Paradox is related to demand changes. The concept of closing some roads implies the corollary of creating new open space in the middle of a city, which can have additional social benefits such as reclaiming land for green spaces, public community centres, landscapes, bikeway, etc. Nonetheless, the side effects of the road closure on the communities and the neighbouring businesses have to be taken into consideration, which is left for further studies. The notion of road closure in this study can also be utilised in developing detour routes for temporary construction in the urban environment. The BPD problem as defined in this study is analogous to what is studied in computer science, for which the results of this study can be useful.

The assumptions made in this study delineate limitation of the study, which can be expanded in further studies. For instance, Braess Paradox in DTA is an open question. In the quest to find Braess-tainted roads (Phase 1), finding a combination of tainted roads is a main challenge that deserves more investigation.

\section{Acknowledgements}

The authors are indebted to three unanimous reviewers whose constructive and insightful comments have contributed to the contents and presentation of the article. The authors thank Dr. Lorna Richardson from Parsons for her helpful comments.

\section{Note}

1. Current Affiliation: Parsons 9123, Dubai, United Arab Emirates.

\section{References}

Aashtiani, H. Z., and H. Poorzahedy. 2004. "Braess' Phenomenon in the Management of Networks and Dissociation of Equilibrium Concepts." Transportation Planning and Technology 27 (6): 469-482.

Abrams, R. A., and J. N. Hagstrom. 2006. "Improving Traffic Flows at No Cost, In Mathematical and Computational Models for Congestion Charging, edited by Lawphongpanich, S., D. W. Hearn, and M. J. Smith, 1-22. New York, USA: Springer.

Akamatsu, T., and B. G. Heydecker. 2003. "Detecting Dynamic Traffic Assignment Capacity Paradoxes in Saturated Networks." Transportation Science 37 (2): 123-138.

Askoura, Y., J. P. Lebacque, and H. Haj-Salem. 2011. "Optimal Sub-Networks in Traffic Assignment Problem and the Braess Paradox". Computers and Industrial Engineering 61 (2): 382-390. 
Arnott, R., A. De Palma, and R. Lindsey. 1993. "Properties of Dynamic Traffic Equilibrium Involving Bottlenecks, Including a Paradox and Metering.” Transportation Science 27 (2): 148-160.

Bagloee, S. A., and M. Tavana. 2012. "An Efficient Hybrid Heuristic Method for Prioritizing Large Transportation Projects with Interdependent Activities." International Journal of Logistics Systems and Management 11 (1): 114-142.

Bagloee, S. A., A. Ceder, and C. Bozic. 2013. "Effectiveness of en route traffic information in developing countries using conventional discrete choice and neural-network models." Journal of Advanced Transportation, available online, DOI: 10.1002/atr.1198.

Bar-Gera, H. (Accessed December 2010). Transportation Network Test Problems, Ben-Gurion University of The Negev, Http://www.Bgu.Ac.Il/ Bargera/Tntp/.

Bazzan, A. L. C., and F. Klügl. 2005. "Reducing the Effects of the Braess Paradox with Information Manipulation." In Applications of Agent Technology in Traffic and Transportation, edited by Klügl et al., 85-98. Basel, Switzerland: Birkhäuser.

Beckman, M. J., C. B. McGuire, and C. B. Winston. 1956. "Studies in the Economics of Transportation." Connecticut : Yale University Press.

Boyce, D. E., H. S. Mahmassani, and A. Nagurney. 2005. "A Retrospective of Beckmann, McGuire, and Winsten's Studies in the Economics of Transportation." Papers in Regional Science 84 (1): 85-103.

Braess, D. 1968. "Über ein Paradoxon aus der Verkehrsplanung." Unternehmensforschung 12: 258-268.

Braess, D., A. Nagurney, and T. Wakolbinger. 2005. "On a Paradox of Traffic Planning." Transportation Science 39 (4): 446-450.

Chen, A., H. Yang, H. K. Lo, and W. H. Tang. 2002. "Capacity Reliability of a Road Network: An Assessment Methodology and Numerical Results." Transportation Research Part B: Methodological, Elsevier 36 (3): 225-252.

Chung, F., and S. J. Young. 2010. "Braess's Paradox in Large Sparse Graphs." In Proceedings of the 6th international conference on Internet and network economics, 194-208. Stanford, CA: Springer-Verlag.

Cole, R., Y. Dodis, and T. Roughgarden, 2006. "How Much Can Taxes Help Selfish Routing?" Journal of Computer and System Sciences 72 (3): 444-467.

Dafermos, S., and A. Nagurney. 1984. "Sensitivity Analysis for the Asymmetric Network Equilibrium Problem." Mathematical Programming 28 (2): 174-184,

Dafermos, S. C., and F. T. Sparrow. 1969. "The traffic assignment problem for a general network." Journal of Research of the National Bureau Standards 72B: 91-118.

Daganzo, C. F. 1998. "Queue spillovers in transportation networks with a route choice." Transportation. Science 32 (1): 3-11.

Fisk, C. 1979. "More Paradoxes in the Equilibrium Assignment Problem." Transportation Research B 13 (4): 305-309.

Hagstrom J. H., and R. A. Abrams. 2001. "Characterizing Braess's Paradox for Traffic Networks." In Proceedings of the fourth IEEE conference on intelligent transportation systems, 837-842.

Hardin, G. 1968. "The Tragedy of the Commons." Science 162 (3859): 1243-1248.

Holland, J. H. 1975. Adaptation in Natural and Artificial Systems. Ann Arbor, MI: University of Michigan Press.

INRO. 2007. “EMME3 User's Guide, Revision 1.22.” Montreal, Quebec, Canada: INRO.

Ipsen, I. C. F., and D. J. Lee. 2006. "Determinant Approximations." Technical Report, Department of Mathematics, North Carolina State University, Raleigh, NC, 2006.

Jenelius, E., 2010, "Large-Scale Road Network Vulnerability Analysis", PhD Dissertation, Department of Transport Science, KTH Royal Institute of Technology, Stockholm, Sweden

Korilis, Y. A., A. A. Lazar, and A. Orda. 1999. "Avoiding the Braess Paradox in Non-Cooperative Networks." Journal of Applied Probability 36 (1): 211-222.

Koutsoupiasm E., and C. Papadimitriou. 1999. "Worst-case equilibria." In 16th annual symposium on theoretical aspects of computer science, 404-413, Trier, Germany, 4-6 March 1999.

Lin, W. H., and H. K. Lo. 2009. “Investigating Braess' Paradox with Time Dependent Queues.” Transportation Science 43 (1): 117-126.

Murchland, J. D. 1970. "Braess's Paradox of Traffic Flow." Transportation Research 4 (4): 391-394.

Nagurney, A. 2010. "The Negation of the Braess Paradox as Demand Increases: The Wisdom of Crowds in Transportation Networks." Europhysics Letters 91 (4): 48002, 1-5.

Nagurney, A., and D. Boyce. 2005. "Preface to On a Paradox of Traffic Planning." Transportation Science 39 (4): 443-445.

Nagurney, A., D. C. Parkes, and P. Daniele. (2007). "The Internet, Evolutionary Variational Inequalities, and the Time-Dependent Braess Paradox." Computational Management Science 4 (4): 355-375. 
Nagurney, A., and Q. Qiang. 2008. “An Efficiency Measure for Dynamic Networks Modeled as Evolutionary Variational Inequalities with Application to the Internet and Vulnerability Analysis." Netnomics, Springer 9 (1): 1-20.

Novak, D. C., J. L. Sullivan, and D. M. Scott. 2012. "A Network-Based Approach for Evaluating and Ranking Transportation Roadway Projects." Applied Geography 34: 498-506.

Park, K. 2011. "Detecting Braess Paradox Based on Stable Dynamics in General Congested Transportation Networks." Networks and Spatial Economics 11 (2): 207-232.

Pas, E. I., and S. L. Principio. 1997. "Braess' Paradox: Some New Insights.” Transportation Research, Series B 31 (3): 265-276.

Pigou, A. C. 1920. "The Economics of Welfare." London: Macmillan.

Poorzahedy, H. and S. N. S. Bushehri. 2005. "Network Performance Improvement under Stochastic Events with Long-Term Effects." Transportation 32 (1): 65-85.

Roughgarden, T. 2001. "Designing Networks for Selfish Users is Hard." In Proceedings of the 42nd annual symposium on foundations of computer science, 472-481. Los Alamitos, CA: IEEE Computer Society Press.

Roughgarden, T. 2002. "The Price of Anarchy is Independent of the Network Topology." In Proceedings of the 34th Annual ACM Symposium on Theory of Computing (STOC), 428-437. Montreal, Canada: ACM Press.

Roughgarden, T. 2005. "Selfish Routing and the Price of Anarchy." Cambridge, MA: MIT Press.

Roughgarden, T. 2006. "On the Severity of Braess's Paradox: Designing Networks for Selfish Users is Hard." Journal of Computer and System Sciences 72 (5): 922-953.

Roughgarden, T., and E. Tardos. 2002. "How Bad is Selfish Routing?" Journal of the ACM 49 (2): 236-259.

Sheffi, Y. 1984. "Urban Transportation Networks: Equilibrium Analysis with Mathematical Programming Methods." New Jersey: Prentice-Hall.

Steinberg, R., and W. I. Zangwill. 1983. "On the Prevalence of the Braess's Paradox.” Transportation Science 17 (3): 301-318.

Stewart, N. F. 1980. "Equilibrium vs. System-Optimal Flow: Some Examples." Transportation Research A 14 (2): 81-84.

Sullivan, J. L., D. C. Novak, L. Aultman-Hall, and D. M. Scott. 2010. "Identifying Critical Road Segments and Measuring System-Wide Robustness in Transportation Networks with Isolating Links: A LinkBased Capacity Reduction Approach." Transportation Research Part A: Policy and Practice 44 (5): 323-336.

Valiant, G., and T. Roughgarden. 2010. "Braess's Paradox in Large Random Graphs." Random Structures and Algorithms 37 (4): 495-515.

Wardrop, J. G. 1952. "Some Theoretical Aspects of Road Traffic Research." Proceedings, Institute of Civil Engineers, PART II, Vol. 1, pp. 325-378.

Yang H., and M. G. H. Bell. 1998. "A Capacity Paradox in Network Design and How to Avoid it." Transportation Research A 32 (7): 539-545.

Yin, Y., and H. Ieda. 2002. "An Optimal Improvement Scheme for Network Reliability." Transportation Research Record 1783: 1-6.

Zhang, X., W. H. K. Lam, and H. J. Huang. 2008. "Braess's Paradoxes in Dynamic Traffic Assignment with Simultaneous Departure Time and Route Choices." Transportmetrica 4 (3): 209-225. 